\title{
Karel Novotný, Neue Konzepte der Phänomenalität
}

Würzburg, Königshausen \& Neumann, 2012, 200 pages

\section{Claudia Serban}

\section{OpenEdition}

\section{Journals}

Édition électronique

URL : http://journals.openedition.org/alter/324

DOI : $10.4000 /$ alter.324

ISSN : 2558-7927

Éditeur :

Association ALTER, Archives Husserl (CNRS-UMR 8547)

\section{Édition imprimée}

Date de publication : 15 novembre 2014

Pagination : 299-304

ISBN : 978-2-9550449-0-2

ISSN : 1249-8947

Référence électronique

Claudia Serban, «Karel Novotný, Neue Konzepte der Phänomenalität », Alter [En ligne], 22 | 2014, mis en ligne le 01 décembre 2017, consulté le 23 septembre 2020. URL : http://journals.openedition.org/alter/ 324 ; DOI : https://doi.org/10.4000/alter.324

Ce document a été généré automatiquement le 23 septembre 2020.

Revue Alter 


\section{Karel Novotný, Neue Konzepte der Phänomenalität}

Würzburg, Königshausen \& Neumann, 2012, 200 pages

Claudia Serban

\section{RÉFÉRENCE}

Karel Novotný, Neue Konzepte der Phänomenalität, Würzburg, Königshausen \& Neumann, 2012, 200 pages. 
Les livres qui essaient de saisir l'unité de la phénoménologie à même ses transformations sont certainement moins nombreux et plus exigeants que les monographies consacrées à tel ou tel auteur ou problème de la tradition phénoménologique. S'inscrivant ouvertement dans le sillage de la grande entreprise de Hans-Dieter Gondek et László Tengelyi dans Neue Phänomenologie in Frankreich (Berlin Suhrkamp, 2011), l'ouvrage de Karel Novotný n'entend cependant pas se limiter aux derniers développements français ou francophones $\mathrm{du}$ mouvement phénoménologique: il s'agit au contraire de trouver une unité dans la multiplicité au sein de ce courant polymorphe, et ce à l'aune d'une question directrice particulièrement puissante et féconde, héritée du phénoménologue tchèque Jan Patočka, dont l'auteur a édité une partie du Nachlass en allemand (Vom Erscheinen als solchem, Fribourg/Munich, Karl Alber, 2000). Cette question est celle de la «nature de l'apparaître » (p.9), et les réponses qui peuvent lui être apportées conduisent à parler de plusieurs et différents « concepts de la phénoménalité ».

2 L'exploration proposée par l'ouvrage commence avec le moment husserlien de la phénoménologie qui est interprété, à travers un prisme patočkien, comme une subjectivation de l'apparaître (chapitre I). L'auteur est néanmoins attentif au sens de l'exigence husserlienne d'un accomplissement (Vollzug) de l'apparaitre sous la forme du vécu (Erlebnis) (p.17), ainsi qu'à la "différence phénoménologique originaire » (p. 23) entre le vécu et le phénomène. La recherche d'un "apparaître pur » (p. 26) exprime alors la tentative de réunir ce qui est vécu et ce qui apparaît dans un seul et même phénomène, et c'est la conscience intime du temps, telle que l'explicite Husserl, qui semble pouvoir répondre à une telle exigence. Mais dans ce cas, la référence de l'apparaître à la subjectivité, loin d'être surmontée, est au contraire renforcée et rendue apodictique. Il s'agit alors d'interroger la portée de cette subjectivation: "l'apparaître chez Husserl est-il lui-même subjectif et, si oui, dans quel sens ?» (p. 30). Cette question confronte cependant aussitôt à un paradoxe, car « ce qui est subjectif dans les phénomènes n'apparaît pas » (p. 35), mais se trouve plutôt au principe de leur apparition. Et la question de la manière dont la subjectivité elle-même se phénoménalise ne reçoit pas non plus de réponse satisfaisante chez Husserl, dans la mesure où pour lui l'ego est ce qu'il est « sans apparition (ohne Erscheinung) » (p. 37).

Le défi est alors précisément d'accorder une véritable teneur phénoménale à la subjectivité censée régir l'apparaître de tout phénomène. C'est ce que l'auteur tente de faire (toujours au chapitre I) en se référant à la phénoménologie matérielle de Michel Henry, qui permet de déterminer le caractère subjectif de l'apparaître comme affectif et impressionnel. Mais cette position qui porte à son comble la subjectivation de 
l'apparaître a aux yeux de l'auteur la faiblesse de rendre tout apparaître impressionnel et donc de désactiver implicitement la différence phénoménologique entre le vécu et ce qui est autre que lui, à savoir la chose qui apparaît. Celle-ci a ses propres modalités d'apparaître, que l'on ne peut pas vraiment décrire comme affectives (pp.43-44): comment interpréter dès lors la thèse selon laquelle toute apparition sensible serait une apparition affective ? Les modalités sensibles de l'apparaître s'annoncent, à l'aune de cette question, comme irréductibles à la manière (impressionnelle et affective) dont le sujet s'apparait et, ce faisant, c'est aussi la tentative d'une subjectivation sans reste de l'apparaître qui s'écroule implicitement.

4 Le chapitre II, consacré à la nature de l'apparaître chez Merleau-Ponty, prend ainsi le contrepied de la tendance à référer intégralement la phénoménalité à la subjectivité, et montre par là que la conjonction entre chair et phénomène peut aussi se décliner en dehors de toute thèse subjectiviste. Il s'agit en outre, pour Merleau-Ponty, de mettre en question la possibilité même d'un " pur phénomène » se trouvant entièrement du côté de la subjectivité et étranger à l'apparaitre mondain (p. 47) : c'est ainsi que l'auteur restitue, dans le cadre qui est le sien, la fameuse assertion de la Phénoménologie de la perception concernant l'impossibilité d'une réduction achevée et complète. Comment saisir et décrire dès lors le champ phénoménal? Le «nouveau concept de la phénoménalité » qui est requis à ce dessein est solidaire d'un "nouveau concept de corps (Leib) » (p. 54) : il s'impose donc de penser la corporéité autrement que comme un principe de subjectivation de l'apparaitre et, ce faisant, de redimensionner la différence phénoménologique entre le vécu et l'apparaissant. L'auteur examine la conception de "l'écart (Abweichung) » qui répond chez Merleau-Ponty à cette nécessité (p. 54 sq.), en tant qu'écart du sujet avec lui-même et de la chair avec elle-même : cet écart est aussi, indissolublement, profondeur, et caractérise à la fois l'apparaître du sujet et l'apparaître du monde. L'écart est donc le « caractère fondamental de la phénoménalité qui, en tant que "chair", est commun au monde en tant que sensible et à la conscience » (p. 66).

5 Au chapitre III, la tentative de penser une autonomie de l'apparaître par rapport à la subjectivité est poursuivie avec un examen du Nachlass de Patočka tout en réintroduisant la référence à Michel Henry en guise de contrepoint. Il s'impose d'abord de restituer la critique pénétrante que le phénoménologue tchèque adresse à la différence phénoménologique entre le vécu et le phénomène telle que Husserl l'explicite et qui ne serait en dernière analyse qu'un "dédoublement artificiel et injustifiable phénoménologiquement de l'apparaître, déterminé essentiellement en tant que mondain, par l'immanence qui ne peut pourtant pas être donnée phénoménalement autrement qu'en tant que mondaine » (p. 69). L'arsenal méthodique d'une épochè sans réduction à l'immanence permet à Patočka de déterminer la sphère phénoménale comme monde et, ce faisant, de poser les bases d'une phénoménologie asubjective qui contrecarre, voire inverse la dissolution du phénomène dans le vécu. La question qui se pose dès lors est celle de savoir comment la chose peut-elle bien être elle-même «l'origine de l'apparaitre (Ursprung des Erscheinens)»(p. 74), question qui permet d'entrevoir la position qui se situe sans doute aux antipodes de celle de Patočka: à savoir, la conception que Michel Henry se fait de la différence phénoménologique comme renvoyant à une dualité de l'apparaître qui est aussi abîme ontologique entre le sujet et le monde. À la détermination patočkienne du champ phénoménal comme monde et à la thèse d'une non-donation immanente de l'ego s'oppose ainsi point par point l'affirmation henryenne d'une indigence ontologique de 
l'apparaître mondain et d'une auto-donation du soi dans l'immanence. À la radicalité sans concession de ces deux positions, l'auteur oppose l'exigence légitime de penser l'apparaître mondain et l'apparaître de la subjectivité dans leur spécificité (p. 89), sans les rabattre systématiquement sur l'autre pôle de la corrélation phénoménologique.

Est-il cependant exact de dire tout d'abord que Patočka aurait omis de penser l'apparaître de la subjectivité ? Le chapitre Iv de l'ouvrage examine le bien-fondé de cette objection en se penchant sur la thématisation patočkienne du corps, de la chair et de l'affectivité à l'intérieur d'une phénoménologie du monde naturel. En tant qu'il est «ce qu'il y a de subjectif dans l'expérience du monde » (p. 96), le corps n'est cependant principe de phénoménalisation que dans la mesure où il est mouvement, et son émotivité (p. 103) renvoie au fait qu'il possède aussi une vie affective propre. Cependant, l'auteur émet un doute quant à la validité qu'aurait cette manière de présenter l'articulation entre apparaître mondain, motricité subjective et vie affective, qui semble confinée par Patočka au cadre de ce qu'il appelle le premier mouvement de l'existence, le seul à être véritablement mû par l'affect et à vivre pleinement dans le corps. À ce stade de l'enquête, la référence au phénoménologue tchèque n'apporte donc pas de réponse satisfaisante à la question directrice de la nature de l'apparaître.

C'est pourquoi l'attention de l'auteur se tourne, au chapitre $\mathrm{v}$, vers la réflexion d'Emmanuel Levinas autour des limites de la phénoménalité, et la reprise critique de la conception husserlienne de la phénoménalité fournit ici encore le point de départ. La mise en avant de la jouissance comme mode sui generis du rapport intentionnel (p. 112 sq.) permet d'envisager autrement - comme nourriture ou comme élément - le corrélat qu'est le phénomène, dont l'apparaître ne peut plus avoir le sens d'une phénoménalisation neutre, à distance, en face du sujet. Cette sortie de la neutralité ou de l'indifférence qui désactive le schéma unilatéral de la donation de sens est à elle seule un premier pas vers le remaniement du sujet en sujet éthique (p. 117). Au seuil de l'éthique cependant, la phénoménalité se dissout devant l'épiphanie du visage, qui fait vaciller la frontière entre ce qui apparaît et ce qui n'apparaît pas. Ainsi, c'est ultimement « autrui qui est principe du phénomène » (p. 124), du véritable phénomène qui renvoie à un mode de révélation incommensurable avec ce qui apparaît communément : l'épiphanie du visage d'autrui est un «apparaître par la dissolution de l'apparaître " (p.126, citant Totalité et infini), apparaître paradoxal de l'infini qui débouche à son tour sur un nouveau concept de la phénoménalité.

8 Mais en deçà de cette épiphanie qui conduit à changer de régime phénoménal, il y a bien chez Levinas une réflexion sur la matérialité et la corporéité de l'apparaitre qui en désignent le moment proprement subjectif, et c'est vers cette réflexion que se tourne le chapitre vI, renouant ainsi avec le fil conducteur des analyses consacrées à Henry et à Patočka. L'auteur relève l'affinité qui existe entre la manière dont Levinas et Henry affirment l'impossibilité d'une récupération intentionnelle du moment proprement impressionnel de la conscience et refusent de traiter le corps propre comme noème ou un objet (p.135). Si Levinas voit dans le corps l'acte et le lieu même de la position du sujet au milieu de l'être impersonnel, il envisage aussi progressivement la dissolution de la matérialité dans la signifiance éthique. Aux yeux de l'auteur, il s'agit là, finalement, d'une dissolution sans reste de la phénoménalité elle-même, qui ne comporte plus de référence à la subjectivité et n'est plus tendue vers aucune épiphanie (p. 141), ce qui vaut aussi, de la part de Levinas, comme un adieu à la phénoménologie. 
Les deux derniers chapitres de l'ouvrage (VII et VIII) sont consacrés aux développements les plus récents de la phénoménologie chez Jean-Luc Marion et Marc Richir, appréhendés toujours à l'aune des transformations qu'ils font subir à la saisie de la phénoménalité. Tout d'abord, l'analyse du principe " autant de réduction, autant de donation" revendiqué et mis en œuvre par Jean-Luc Marion permet de retrouver l'exigence de penser l'autonomie de l'apparaître : il suffit que le phénomène se donne pour qu'il apparaisse (p. 146) ; sa phénoménalisation ne dépend d'aucune prestation de la subjectivité. L'auteur interroge alors le statut de la donation elle-même, qui ne semble pas pouvoir se donner elle-même dans le phénomène qui apparaît et, ce faisant, nous conduit encore une fois devant les limites de la phénoménologie, qui coïncident avec les limites de la phénoménalité (p. 153). Alors que l'universalisation de la donation devait permettre d'affirmer que tout doit pouvoir apparaître, le fait que la donation ellemême se soustrait à la phénoménalité conduit à retrouver le paradoxe qui veut que «le principe des phénomènes demeure au-delà de la phénoménalité » (p.156); autrement dit : « la limite de la phénoménalité est dans son principe » (p. 161).

Peut-on reposer la question de la phénoménalisation sans aboutir nécessairement à un tel paradoxe ? Le dernier chapitre (viII) de l'ouvrage relève ce défi en s'attaquant aux recherches phénoménologiques de Marc Richir, animées de part en part par le problème de la nature de l'apparaitre. La visée d'une "défénestration» (p.167) de l'apparaître chez le philosophe belge rejoint les critiques de l'excessive subjectivation de la phénoménalité sans pour autant rabattre intégralement l'apparaître et son principe du côté du monde. La mise au jour d'un apparaître tout autant pré-mondain et pré-subjectif s'accompagne d'une compréhension du phénomène comme «sens symboliquement institué " et de la phénoménalisation comme "formation de sens spontanée» (p.169). En même temps, cette conception de l'apparaître revient à reconnaître une négativité fondamentale au cœur du phénomène, qui a partie liée avec le rien et n'est en conséquence «rien que phénomène " (p. 171). L'auteur voit « une version radicalisée de la phénoménologie » (p. 174) dans cette conception selon laquelle la phénoménalisation est constitutivement tendue entre l'apparition et la disparition et consiste essentiellement dans une oscillation de l'apparaître ou dans un clignotement.

11 Nous pouvons remarquer pour notre part, en déplorant le manque d'une conclusion générale de l'ouvrage ou du moins d'une mise en commun et d'une confrontation des acquis des deux derniers chapitres, que l'alternative devant laquelle la phénoménalité semble se trouver désormais n'est plus celle entre le sujet et le monde, mais celle située en deçà du sujet comme en deçà du monde - entre la donation et la non-donation du phénomène. Mais s'il est sans doute impossible de conclure sur ce qui n'est pas encore achevé, le chemin sinueux des transformations de la phénoménologie, traversé par l'auteur en prenant pour guide la question de la nature de l'apparaître, devient à coup sûr, au terme de l'ouvrage, mieux éclairé et donc plus praticable. 